\title{
Radiotherapy of glioblastoma 15 years after the landmark Stupp's trial: more controversies than standards?
}

\author{
Tomas Kazda1,2,3, Adam Dziacky ${ }^{4}$, Petr Burkon ${ }^{1,2}$, Petr Pospisil' ${ }^{1,2}$, Marek Slavik ${ }^{1,2}$, \\ Zdenek Rehak ${ }^{5,6,7,8}$, Radim Jancalek ${ }^{9,10}$, Pavel Slampa ${ }^{1,2,6}$, Ondrej Slaby, ${ }^{3,7,8}$, \\ Radek Lakomy 7,8

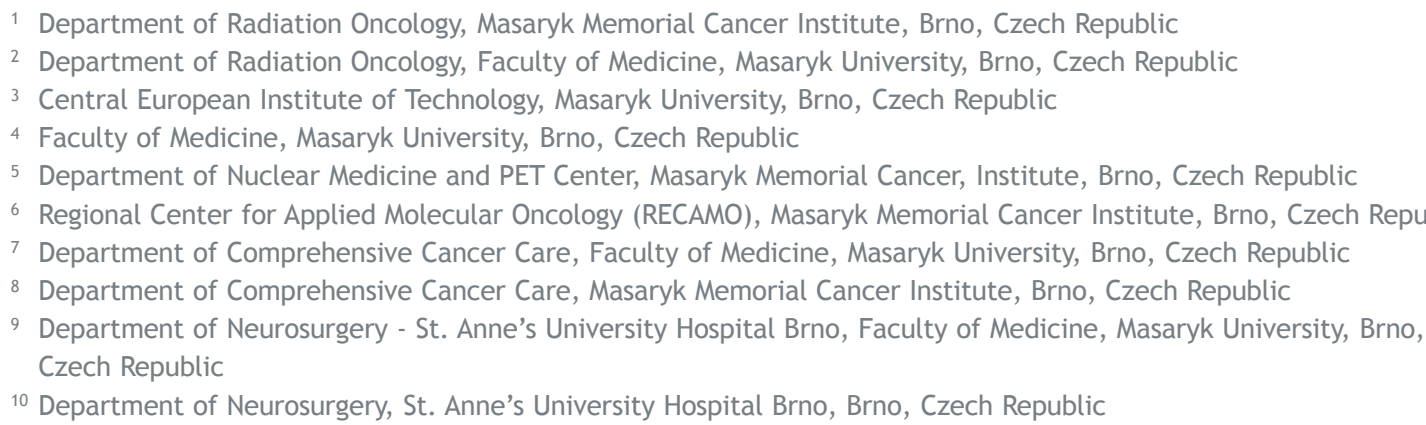

Background. The current standard of care of glioblastoma, the most common primary brain tumor in adults, has remained unchanged for over a decade. Nevertheless, some improvements in patient outcomes have occurred as a consequence of modern surgery, improved radiotherapy and up-to-date management of toxicity. Patients from control arms (receiving standard concurrent chemoradiotherapy and adjuvant chemotherapy with temozolomide) of recent clinical trials achieve better outcomes compared to the median survival of 14.6 months reported in Stupp's landmark clinical trial in 2005. The approach to radiotherapy that emerged from Stupp's trial, which continues to be a basis for the current standard of care, is no longer applicable and there is a need to develop updated guidelines for radiotherapy within the daily clinical practice that address or at least acknowledge existing controversies in the planning of radiotherapy.

The goal of this review is to provoke critical thinking about potentially controversial aspects in the radiotherapy of glioblastoma, including among others the issue of target definitions, simultaneously integrated boost technique, and hippocampal sparing.

Conclusions. In conjunction with new treatment approaches such as tumor-treating fields (TTF) and immunotherapy, the role of adjuvant radiotherapy will be further defined. The personalized approach in daily radiotherapy practice is enabled with modern radiotherapy systems.

Key words: glioblastoma; radiation therapy; controversy; target volumes; radiotherapy dosage

\section{Introduction}

Despite many advances in the understanding of glioblastoma (GBM) biology in recent decades, only a few findings were translated into updates in the treatment guidelines for this most aggressive and frequent primary brain tumor of adults. These updates have notably occurred in the manage- 
ment of elderly patients, where methylation of the O6-methylguanine-DNA methyltransferase (MGMT) gene promotor indicates higher chemosensitivity and higher benefit from administration of alkylating agents such as temozolomide (TMZ). ${ }^{1-5}$ Unlike for the majority of other cancer types in which there have been treatment gains with the advent of targeted therapies, there have not been similar advances in GBM treatment to date. Owing to rich tumor neovascularization, much hope was put especially into anti-angiogenic therapy ${ }^{6}$; however, negative results have been reported in GBM clinical trials that used bevacizumab to target vascular endothelial growth factor., ${ }^{7,8}$ In contrast with negative results from recent GBM trials focused on new pharmacotherapeutics, co-administration of RT with TMZ has nearly tripled the 2-year survival of GBM patients in the last decade from a dismal $10 \%$ with RT alone to $27 \%$ with the addition of TMZ and quintupled to $47 \%$ in patients with MGMT promoter methylation ${ }^{9}$, representing an exciting advance after little progress in previous decades. ${ }^{10}$ However, standard post-surgery treatment of newly diagnosed GBM patients has remained unchanged since implementation of the recommendations of the EORTC 26981-22981/NCIC CE3 trial (Stupp regimen) that finished enrolling patients in 2002 and was published in 2005.9,11 In this protocol, TMZ $\left(75 \mathrm{mg} / \mathrm{m}^{2}\right)$ is administered on days 1 through 42 with concomitant RT (60 Gy), followed by administration of TMZ alone (150 to $200 \mathrm{mg}$ / $\mathrm{m} 2$ ) on days 1-5 in six consecutive 4 -week cycles. Co-administration of TMZ improved survival from 12.1 months (with RT alone) to 14.6 months (with the addition of TMZ). ${ }^{11,12}$

This educational review considers potentially controversial aspects in the RT of GBM assuming strict application of the current standard of care EORTC 26981-22981/NCIC CE3 protocol for RT planning.

\section{Current updates in glioblastoma treatment}

Despite the advance described above, patients in ordinary clinical practice (outside of clinical trials) have now been treated with the same general protocol for more than 10 years. During that time, though, RT itself has experienced rapid evolution due to advances in computing technology, better access to imaging methods, and more sophisticated RT instrumentation. ${ }^{13}$ Today, preparation and application of RT is much more complicated than 10 years ago, but does this complexity bring any benefits in regards to overall survival of patients with GBM? Even though patients receive a numerically identical dose of $60 \mathrm{~Gy}$, the dosing technique matters as it affects toxicity. There is an obvious difference between conventional 2-dimensional external beam RT (as whole brain irradiation, WBRT) and the 3-dimensional conformal RT or other modern methods of photon RT (e.g., intensity-modulated arc therapy, IMRT). Recently, tumor-treating fields (TTFs) have become recognized as a novel cancer treatment modality with antimitotic effects against rapidly dividing tumor cells. ${ }^{14}$ This is caused by alternating electric fields of low-intensity and intermediate-frequency through transducer arrays applied to the shaved head, which are being increasingly thought of as an upcoming new standard of care in GBM, already approved by the U.S. Food and Drug Administration for both newly diagnosed as well as recurrent GBM. ${ }^{15}$ A randomized clinical phase 3 trial EF-14 evaluated the effect of TTF plus maintenance TMZ vs. maintenance TMZ alone on survival parameters in patients with newly diagnosed GMB. ${ }^{16,17}$ This trial represents the first major advance in the treatment of newly diagnosed GBM in roughly a decade, with a hazard ratio for overall survival of 0.63 being numerically comparable with that seen in the Stupp trial in 2005. Ultimately, aside from health-care payers' points of view, the willingness of patients to undergo the burden of carrying a TTF device non-stop will determine if TTF becomes a new standard of care. ${ }^{18}$ Based on the interim analysis, there is no preliminary evidence that health-related quality of life, cognitive, or functional status is adversely affected by continuous usage of TTF. ${ }^{19}$

Notably, patients in control (standard therapy) arm of this trial achieved relatively long median survival, as well as in another recent trial (ACT IV trial), where the role of epidermal growth factor receptor EGFRvIII targeted vaccine-based immunotherapy rindopepimut was investigated. ${ }^{20}$ Compared to a median overall survival (OS) of 14.6 months in the original Stupp trial ${ }^{11}$, there was reported to be significant increase in median OS (from diagnosis to death) to 19.8 months in the EF14 trial $^{17}$, and to 20.2 months (median 17.4 months from randomization to death + a reported median of 2.8 months from diagnosis to randomization) in the control arm of the rindopepimut ACT IV trial. ${ }^{20}$ Since the underlying treatment (RT to $60 \mathrm{~Gy}+$ concomitant and maintenance TMZ) is the same, it is unclear whether this difference in median OS is the evidence of improved treatment outcomes as 
a consequence of modern surgery, improved RT, and up-to-date management of toxicity. Patients in both of these studies were randomized after the completion of concomitant chemoradiotherapy. Thus, more favorable patients were selected compared to the first landmark Stupp trial. For this reason, a selection bias may be responsible for the described difference in OS. 82 out of 1019 (8\%) eligible patients for EF-14 trial experienced progressive disease after completion of radiotherapy phase and were excluded. ${ }^{17}$ Whether $8 \%$ patients with the worst prognosis would have been excluded in EORTC 26981-22981/NCIC CE3 trial, the median overall survival would be roughly 16.5 months from randomization (17.7 months from diagnosis). So without eventual selection bias, it is possible to compare these 17.7 months in the Stupp trial to the 19.8 months in EF-14 and 20.2 months in rindopepimut trials, all with the same treatment $\mathrm{RT}+\mathrm{TMZ}$. The difference in more than 2 months is clear improvement in outcomes as a consequence of modern surgery, RT and toxicity management. Or should we utilize approaches in daily RT practice which were employed in the Stupp trial decade ago since this is a "registration" trial for the current standard of care?

\section{The correct total dose}

Despite advances in RT over the last 10 to 15 years, certain postulates remain unchanged. One such principle is: "the correct dose to the correct place." Dosage for adjuvant RT of GBM has been same over the last few decades, and it is not typically considered controversial in patients younger than 60-65 years. ${ }^{21}$ The effect of high doses in adjuvant RT of GBM was shown in 1979 by a retrospective analysis performed by the German "Brain Tumor Study Group". ${ }^{23}$ The best result (median overall survival of 42 weeks) was achieved by WBRT with 60 Gy compared to 55 (36 weeks) or 50 Gy (28 weeks) $)^{23}$ and with significant difference between those receiving 60 versus $50 \mathrm{~Gy}$. Doses above 60 Gy did not lead to any benefit regardless of RT technique used. With WBRT, increasing to $70 \mathrm{~Gy}$ was not associated with further survival improvement. ${ }^{24}$ The later attempts to improve outcomes by an increase of RT dose included combination of 60 Gy WBRT and increased targeted dosage by IMRT $^{25}$, brachytherapy ${ }^{26}$, or stereotactic radiosurgery (RTOG 9305). ${ }^{27}$ Increasing focused radiation dose by hyperfractionation was tested as well. ${ }^{28}$ Doses above $60 \mathrm{~Gy}$ have not proven to be beneficial even in the TMZ era. ${ }^{29}$
One might conclude that the question of the correct total dose in adjuvant RT is a closed chapter and further studies in this field are not judged. On the other hand, it is possible that the studies mentioned above missed application of increased RT dose to the most malignant tumor cells (a highdensity portion of tumor). Such areas are generally considered to be areas with contrast enhancement on CT or MRI. MRI-guided serial biopsy study has proven that tumor cells are present inside but also outside the area of this enhancement, infiltrating at least borders of T2 hyperintensity on MRI. ${ }^{30}$ Positron emission tomography (PET) has an increasingly important role in the diagnosis, grading, response assessment, and/or guidance of surgery and RT. ${ }^{31-33}$ For example, ${ }^{18}$ F-DOPA (3,4-dihydroxy$6-\left[{ }^{18} \mathrm{~F}\right]$ fluoro-1-phenylalanine) is an amino acid tracer that identifies areas of high-grade portions of disease as proven by histopathology evaluation of ${ }^{18} \mathrm{~F}$-DOPA and MRI-guided biopsies with statistically significant difference in tumor-to-normal brain uptake ratio between grade II, III and IV gliomas. ${ }^{34}$ Dose escalation to not only areas with postcontrast MRI enhancement but also to high-risk areas identified by PET or diffusion/perfusion MRI may improve clinical outcomes of glioma treatment. ${ }^{35}$ An ongoing phase II clinical trial for highgrade gliomas is evaluating increases in the dose up to 76 Gy with target volume defined by MRI and ${ }^{18} \mathrm{~F}-\mathrm{DOPA}$ PET (NCT01991977). Dose-escalated proton beam RT is being evaluated in an ongoing prospective trial as well (NCT02179086). Hence, even if the current RT dosage is well established at $60 \mathrm{~Gy}$ in common standard of care, the correct dose of radiation may become controversial shortly with wider availability of advanced MR and PET imaging or proton beam facilities. ${ }^{36,37}$ Furthermore, as we gain additional experience with TTF therapy, effects of concurrent administration of TTF and RT will be questioned, and dose escalation or de-escalation trials may be of substantial interest in the field, further clouding the issue of correct RT dose.

\section{The correct RT target definition}

Determination of the ideal target volume for RT represents a trade-off between minimizing treatment-related toxicity and achieving tumor control. With standard structural MRI, there are several comparable methods of contouring clinical target volume (CTV: location of expected or suspected malignant cells). Two basic approaches in target definition are "the American approach" by the Radiation Therapy Oncology Group (RTOG con- 
TABLE 1. Recommendations for target definition according to EORTC, RTOG and ESTRO-ACROP

\begin{tabular}{|c|c|c|c|}
\hline $\begin{array}{l}\text { Contouring } \\
\text { approach }\end{array}$ & Dose prescription & GTV & CTV \\
\hline $\begin{array}{l}\text { EORTC } \\
\text { single phase }\end{array}$ & 30 x 2.0 Gy & $\begin{array}{l}\text { Resection cavity } \\
+ \text { residual Tl enhancement }\end{array}$ & $\mathrm{GTV}+2 \mathrm{~cm}$ \\
\hline \multirow[t]{2}{*}{$\begin{array}{l}\text { RTOG } \\
\text { two phases }\end{array}$} & 23 x $2.0 \mathrm{~Gy}$ & $\begin{array}{l}\text { GTV1: } \\
\text { Resection cavity } \\
\text { + residual T1 enhancement } \\
\text { + FLAIR abnormality (oedema) }\end{array}$ & $\begin{array}{l}\mathrm{CTV} 1=\mathrm{GTV} 1+2 \mathrm{~cm} \text { (the margin is } \\
2.5 \mathrm{~cm} \text { in cases where no oedema } \\
\text { is presented) }\end{array}$ \\
\hline & $+7 \times 2.0 \mathrm{~Gy}$ & $\begin{array}{l}\text { GTV2: } \\
\text { Resection cavity } \\
\text { + residual T1 enhancement }\end{array}$ & $\mathrm{GTV} 2+2 \mathrm{~cm}$ \\
\hline ESTRO-ACROP & 30 x $2.0 \mathrm{~Gy}$ & $\begin{array}{l}\text { Resection cavity } \\
\text { + residual Tl enhancement } \\
\text { + FLAIR abnormality (oedema) } \\
\text { for secondary glioblasomas }\end{array}$ & $\mathrm{GTV}+2 \mathrm{~cm}$ \\
\hline
\end{tabular}

Abbreviations: EORTC = European Organisation for Research and Treatment of Cancer; ESTRO-ACROP = European Society for Radiotherapy \& Oncology - Advisory Committee on Radiation Oncology Practice; CTV = clinical target volume; FLAIR = Fluid-attenuated Inversion Recovery. GTV = gross tumor volume; RTOG = Radiation Therapy Oncology Group

touring approach) that defines two CTVs accommodating hyperintensity at T2/FLAIR MRI (FLAIR Fluid-attenuated Inversion Recovery) in addition to $\mathrm{T} 1$ contrast-enhanced $\mathrm{MRI}^{38}$ and "the European approach" by the European Organization for Research and Treatment of Cancer (EORTC single phase contouring approach) that defines one CTV utilizing mainly T1 post-contrast MRI. The ESTROACROP (European Society for Radiotherapy \& Oncology - Advisory Committee on Radiation Oncology Practice) approach resembles EORTC practice, although CTV is defined in certain instances also by T2/FLAIR MRI, especially in the case of secondary, isocitrate dehydrogenase (IDH)mutated $\mathrm{GBM}^{39}$ (Table 1). There has been no randomized comparison of these different consensus practices. Nevertheless, the single phase approach is generally associated with reduced irradiated volume without a significant increase in marginal or distant recurrences. ${ }^{40}$ Reducing target volumes leads to lower irradiation of radiographically normal brain and, thus, possibly to less toxicity, although this remains to be validated prospectively.

In daily clinical practice, the chosen method for contouring depends in part on the planned radiation technique (3-dimensional conformal therapy vs. inverse planning of IMRT with steeper dose gradients and higher demand on precision) or on the extent of edema, respective of the tumor itself. Molecular characteristics of gliomas, currently established in the integral diagnosis within the new WHO classification update from 2016, may also influence target definition in the personalized contouring of target volumes. ${ }^{41}$ Mutation of the IDH gene, the early stable driving mutation in diffuse glioma, is associated with WHO grade II/
III gliomas and a better prognosis, while GBMs are typically IDH wild-type. Nonetheless, about 10\% of GBMs, formerly named secondary GBMs, are IDH-mutated, which probably indicates their dedifferentiation from low-grade gliomas. Because this dedifferentiation may occur anywhere within initial low-grade glioma, a single phase approach with high dose irradiation covering all T2/FLAIR hyperintensity may be deemed suitable. Nevertheless, it is too soon to speak about a predictive marker for RT because no clinical trial or RT planning study so far addressed this issue.

If a two-phase RTOG strategy is applied for a particular patient, the dose for "the larger volume" and the dose for "the smaller volume - the highrisk region" must be determined. This cone-down strategy, also called sequential boost, combines most often $46+14 \mathrm{~Gy}$, or possibly $50+10 \mathrm{~Gy}$, depending on target volume size. IMRT allows preparation of irradiation for two target volumes simultaneously. This technique, called simultaneous integrated boost (SIB), is sometimes used in clinical practice. However, its usage may be controversial. A common prescription is $30 \times 1.7 \mathrm{~Gy}$ (51 Gy) for planning target volume \#1 and 30 × $2.0 \mathrm{~Gy}$ (60 Gy) for planning target volume \#2. This is not truly an SIB, because the dose in high-risk subvolume does not exceed standard daily 2.0 Gy; instead, the dose in low-risk subvolume is decreased to daily $1.7 \mathrm{~Gy}$. The advantage is in dose control within individual subvolumes. In the case of the standard sequential 46 + 14 Gy regimen, the low-risk subvolume receives more than the prescribed 46 Gy (Figure 1) because irradiation during the second phase passes through the low-risk subvolume (resulting in a nonhomogeneous dose of about 50-57 Gy, of which 
first 46 Gy is delivered in 2.0 Gy daily fractions). As long as all dose-volume constraints are met, such an RT plan can be clinically applied. SIB seems to be more beneficial for cases with a large volume of hyperintensity on T2/FLAIR-weighted MRI, because the total dose for low-risk subvolume exactly matches the prescribed dose. Assuming relative radioresistance of glioma cells, the disadvantage is in the low daily dose of 1.7 Gy. There are no clinical studies that prove or disprove superiority of this type of SIB, and therefore its use remains controversial. However, there are also no robust clinical studies demonstrating benefits of IMRT in general. ${ }^{42}$ Some reports exist in the evaluation of classical SIB, where the RT schedule is hypofractionated. For example, Paner-Raymond reported no survival improvement or patterns of failure change in their retrospective analysis of patients treated in 20 fraction of 2.0 Gy delivered to the larger volume while simultaneously boosting gross tumor volume by 3.0 Gy. ${ }^{43}$ The same RT protocol was used in recently published phase II clinical trial evaluating the role of neoadjuvant TMZ. ${ }^{4}$ Hypofracionated (60 Gy in 20 daily fractions) RT was administrated concurrently with TMZ after 2 weeks of prior neoadjuvant TMZ (75 mg/m² per day) and was followed by adjuvant TMZ. ${ }^{44}$ Encouraging median overall survival of 22.3 months warrants further testing of this approach in phase III design. Until then, hypofractionated SIB cannot be considered a standard for daily clinical practice. Generally, there is potential for a planning study and then even maybe a trial (comparison of RTOG vs. EORTC contouring approaches, normofractionated or hypofractionated SIB techniques vs. cone-down strategy, etc), however only one topic at the time must be addressed.

IMRT is commonly used due to its apparent dosimetric advantages, mainly for tumors localized close to critical organs. The question often asked about expensive particle therapy is also pertinent for IMRT: What evidence do we need for the establishment of a new standard of care in RT techniques? Dosimetric advantages have the potential to translate into the better neurocognitive function as is currently evaluated in the NCT01854554 clinical trial, where IMRT is compared with intensitymodulated proton RT for newly diagnosed GBM.

\section{Lessons from the past: RT approach from Stupp's protocol}

The RT planning steps in the original Stupp's protocol were substantially less complicated than those used nowadays. Gross tumor volume (GTV)

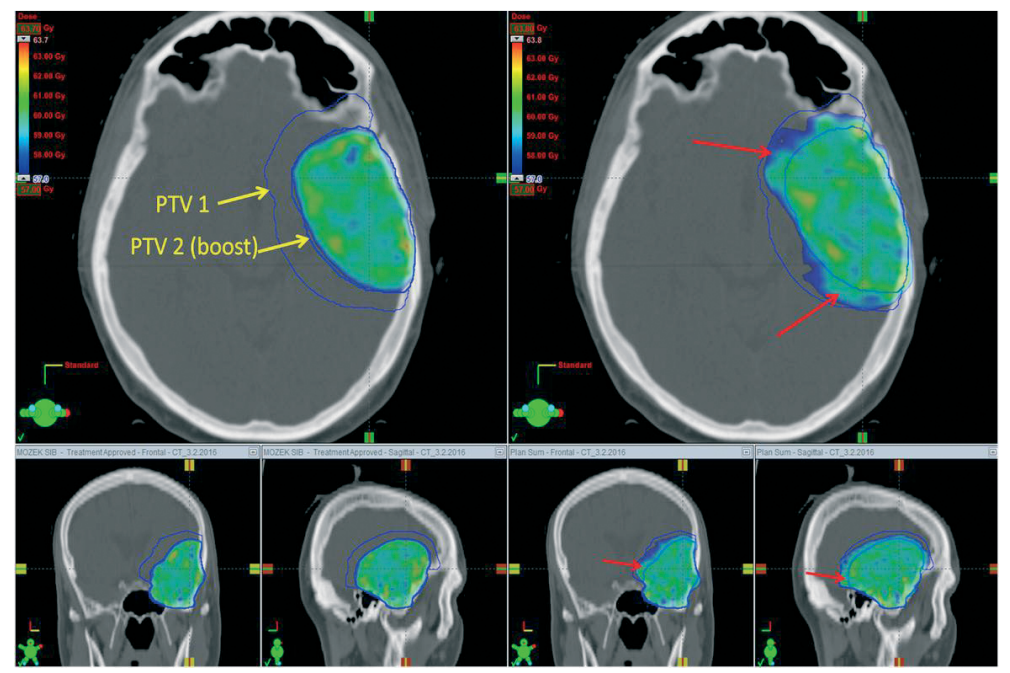

FIGURE 1. An example of an RT treatment plan (color wash display of isodoses with a minimal dose of $57 \mathrm{~Gy}$, that is $95 \%$ of the prescribed dose of $60 \mathrm{~Gy}$ ) in three planes. A RT plan for the same patient was prepared using a simultaneous integrated boost (left) and sequential boost (right). Target volumes are shown in blue contour (yellow labeled arrows). Dose assignment to the "PTV2-boost" target volume is the same in boost cases, $30 \times 2.0 \mathrm{~Gy}$. With sequential boost, overtreatment (red arrows) is observed in the area where a lower dose was prescribed.

representing tumor mass, was defined as the area within the primary tumor as measured by post-contrast enhancement on either CT or MRI. In general terms, planning target volume (PTV: margin needed to compensate for inaccuracies) is the GTV enlarged by approximately $2-3 \mathrm{~cm} .{ }^{11}$ Determination of a safety margin for PTV is complicated in daily clinical practice with a slight difference in each patient as several variables must be taken into consideration. The most important are planned technique (classical conformal RT vs. IMRT; coplanar vs. non-coplanar), quality of immobilization, availability of on-board imaging system, inaccuracy in images registration, compliance, and overall patient's state.

Based on the evaluation of randomly selected individual patients from all participating centers in the EORTC 26981-22981/NCIC CE3 trial, 34\% of centers planned RT based solely on pre-operative $\mathrm{CT}$ and $62 \%$ of centers planned RT on classic 2-dimensional simulator measurements. ${ }^{45}$ Most of the centers (94\%) delineated PTV alone or with CTV or GTV. ${ }^{45}$ Would that be considered as a lege artis or as a controversial, whether we plan RT for our patient on 2 dimensional RTG simulator without dedicated planning CT (and MRI) scan, since it was employed in a registry study of the current standard of care? 


\section{Other controversies in the decision- making of daily RT practice}

A few other controversies associated with RT of HGG are mentioned briefly. The issue of reduction of CTV around natural barriers to tumor growth is not standardized. One of the options is to reduce the CTV to as low as zero at the border with fixed barriers such as bone or falx and to as low as few millimeters around non-rigid barriers such as ventricles or brain stem. Another controversial topic is hippocampal sparing with the goal of minimizing the negative effects of RT on cognitive functions. ${ }^{46,47} \mathrm{RT}$ in $2 \mathrm{~Gy}$ fractions to $40 \%$ of the bilateral hippocampi greater than an equivalent dose of 7.3 Gy was associated with long-term memory impairment in patients with low-grade or benign brain tumors. ${ }^{47}$ There is controversy as to whether it might or might not be beneficial to spare the ipsilateral neural stem cell regions as in the hippocampus, owing to accumulating evidence of higher risk of tumor recurrence in the proximity to these regions, which are thought to provide favorable conditions for putative glioma stem cells whose survival is believed to be responsible for tumor recurrence. ${ }^{48-50}$ Indeed, subventricular zones have been proposed as new key targets for GBM treatment. ${ }^{51,52}$ Whether to spare the contralateral hippocampus remains controversial. Nevertheless, especially when employing inverse RT planning to allow dose control in different parts of the brain, contralateral hippocampal sparing should be at least considered in patients with right-sided low-grade glioma to spare the dominant left hemisphere which may be more related to verbal memory declines after RT. 53,54

\section{Conclusion and future perceptivities}

To provide state-of-the-art RT for GBM patients in daily clinical practice, it is necessary to acknowledge the capabilities and limitations of current RT techniques in the light of the potential controversies described in this review. With current technical and supportive care advances, it is possible to prolong further the survival of patients with GBM as presented in the control arms of the recent EF-14 and rindopepimut ACT IV trials. In trials enrolling patients who have completed chemoradiotherapy, significant differences in completed upfront treatment may affect results; further confounding influences can be at least partially mitigated if the above-mentioned uncertainties and controversies in daily RT are used as stratifications factors. For example, for the EF-14 trial, trial protocol amendment V2.0 included an update in the dose of RT (from 60 Gy to 45-70 Gy) to address common variations in the standard of care treatment between individual patients/centers. ${ }^{16}$ Although the proportion of patients who received less than $57 \mathrm{~Gy}$ was balanced between the TTF and control arms, one must ask what kind of RT should be used in daily clinical practice if the TTF treatment were to become the new standard of care. With the current standard approach of concomitant and adjuvant $\mathrm{TMZ}$, it might not matter which RT procedure is chosen. With new treatment approaches such as TTF or immunotherapy ${ }^{55-57}$, the role of adjuvant radiotherapy will be further defined.

\section{Acknowledgments and funding}

This work was supported in part by the Ministry of Health, Czech Republic - Conceptual Development of Research Organization (MMCI 00209805) and project MEYS-NPS I-LO1413. The results of this research have been acquired within CEITEC 2020 (LQ1601) project with the financial contribution made by the Ministry of Education, Youths and Sports of the Czech Republic within special support paid from the National Programme for Sustainability II funds.

\section{Statement}

The paper has not been published or submitted for publication elsewhere. This is the first submission of this paper. All listed authors concur in the submission and are responsible for its content. They agree with publication and have given the corresponding author the authority to act on their behalf in all matters pertaining to publication.

\section{References}

1. Roa W, Brasher PM, Bauman G, Anthes M, Bruera E, Chan A, et al. Abbreviated course of radiation therapy in older patients with glioblastoma multiforme: a prospective randomized clinical trial. J Clin Oncol 2004; 22: 1583-8. doi: 10.1200/JCO.2004.06.082

2. Malmström A, Grønberg BH, Marosi C, Stupp R, Frappaz D, Schultz H, et al. Temozolomide versus standard 6-week radiotherapy versus hypofractionated radiotherapy in patients older than 60 years with glioblastoma: the Nordic randomised, phase 3 trial. Lancet Oncol 2012; 13: 916-26. doi: 10.1016/S1470-2045(12)70265-6

3. Wick W, Platten M, Meisner C, Felsberg J, Tabatabai G, Simon M, et al. Temozolomide chemotherapy alone versus radiotherapy alone for malignant astrocytoma in the elderly: The NOA-08 randomised, phase 3 trial. Lancet Oncol 2012; 13: 707-15. doi: 10.1016/S1470-2045(12)70164-X

4. Perry JR, Laperriere N, O'Callaghan CJ, Brandes AA, Menten J, Phillips C, et al. Short-course radiation plus temozolomide in elderly patients with glioblastoma. N Engl J Med 2017; 376: 1027-37. doi: 10.1056/NEJMoa1611977 
5. Hegi ME, Diserens AC, Gorlia T, Hamou MF, de Tribolet N, Weller M, et al. MGMT gene silencing and benefit from temozolomide in glioblastoma. $N$ Engl J Med 2005; 352: 997-1003.

6. Wick W, Platten M, Wick A, Hertenstein A, Radbruch A, Bendszus M, et al. Current status and future directions of anti-angiogenic therapy for gliomas. Neuro Oncol 2016; 18: 315-28. doi: 10.1093/neuonc/nov180

7. Chinot OL, Wick W, Mason W, Henriksson R, Saran F, Nishikawa R, et al. Bevacizumab plus radiotherapy-temozolomide for newly diagnosed glioblastoma. N Engl J Med 2014; 370: 709-22. doi: 10.1056/NEJMoa1308345

8. Gilbert MR, Dignam JJ, Armstrong TS, Wefel JS, Blumenthal DT, Vogelbaum $\mathrm{MA}$, et al. A Randomized trial of bevacizumab for newly diagnosed glioblastoma. N Engl J Med 2014; 370: 699-708. doi: 10.1056/NEJMoa1308573

9. Stupp R, Hegi ME, Mason WP, van den Bent MJ, Taphoorn MJB, Janzer RC, et al. Effects of radiotherapy with concomitant and adjuvant temozolomide versus radiotherapy alone on survival in glioblastoma in a randomised phase III study: 5-year analysis of the EORTC-NCIC trial. Lancet Oncol 2009; 10: 459-66. doi: 10.1016/S1470-2045(09)70025-7

10. Uhm JH, Porter AB. Treatment of Glioma in the 21st Century: An exciting decade of postsurgical treatment advances in the molecular era. Mayo Clin Proc 2017; 92: 995-1004. doi: 10.1016/j.mayocp.2017.01.010

11. Stupp R, Mason WP, van den Bent MJ, Weller M, Fisher B, Taphoorn MJB, et al. Radiotherapy plus concomitant and adjuvant temozolomide for glioblastoma. N Engl J Med 2005; 352: 987-96. doi: 10.1056/NEJMoa043330

12. Alexander BM, Cloughesy TF. Adult glioblastoma. J Clin Oncol 2017; 35: 2402-09. doi: 10.1200/JCO.2017.73.0119

13. Halasz BLM, Soltys SG, Breneman JC, Chan MD, Laack NN, Minniti G, et al. Treatment of gliomas: a changing landscape. Int J Radiat Oncol Biol Phys 2017; 98: 255-8. doi: 10.1016/j.jijrobp.2017.02.223

14. Hottinger AF, Pacheco P, Stupp R. Tumor treating fields: a novel treatment modality and its use in brain tumors. Neuro Oncol 2016; 18: 1338-49. doi: 10.1093/neuonc/now182

15. Mehta M, Wen P, Nishikawa R, Reardon D, Peters K. Critical Reviews in Oncology / Hematology Critical review of the addition of tumor treating fields (TTFields) to the existing standard of care for newly diagnosed glioblastoma patients. Crit Rev Oncol Hematol 2017; 111: 60-5. doi: 10.1016/j. critrevonc.2017.01.005

16. Stupp R, Taillibert S, Kanner AA, Kesari S, Steinberg DM, Toms SA, et al. Maintenance therapy with tumor-treating fields plus temozolomide vs temozolomide alone for glioblastoma. JAMA 2015; 314: 2535-43. doi: 10.1001/jama.2015.16669

17. Stupp R, Taillibert S, Kanner A, Read W, Steinberg DM, Lhermitte B, et al. Effect of Tumor-treating fields plus maintenance temozolomide vs maintenance temozolomide alone on survival in patients with glioblastoma: A randomized clinical trial. JAMA 2017; 318: 2306-16. doi: 10.1001/ jama.2017.18718

18. Wick W. TTFields: Where does all the skepticism come from? Neuro Oncol 2016; 18: 303-5. doi: 10.1093/neuonc/now012

19. Zhu JJ, Demireva P, Kanner AA, Pannullo S, Mehdorn M, Avgeropoulos $\mathrm{N}$, et al. Health-related quality of life, cognitive screening, and functional status in a randomized phase III trial (EF-14) of tumor treating fields with temozolomide compared to temozolomide alone in newly diagnosed glioblastoma. J Neurooncol 2017 Aug 28. doi: 10.1007/s11060-017-2601-y. [Epub ahead of print]

20. Weller M, Butowski N, Tran DD, Recht LD, Lim M, Hirte H, et al. Rindopepimut with temozolomide for patients with newly diagnosed, EGFRvIll-expressing glioblastoma (ACT IV): a randomised, double-blind, international phase 3 trial. Lancet Oncol 2017; 18: 1373-85. doi: 10.1016/S1470-2045(17)30517-X

21. Corso CD, Bindra RS, Mehta MP. The role of radiation in treating glioblastoma: here to stay. J Neurooncol 2017 Mar 7. doi: 10.1007/s11060-0162348-x. [Epub ahead of print]

22. Roth $P$, Gramatzki $D$, Weller M. Management of elderly patients with glioblastoma. Curr Neurol Neurosci Rep 2017; 17: 35. doi: 10.1007/s11910017-0740-3

23. Walker MD, Strike TA, Sheline GE. An analysis of dose-effect relationship in the radiotherapy of malignant gliomas. Int J Radiat Oncol Biol Phys 1979; 5: 1725-31. doi: 10.1016/0360-3016(79)90553-4
24. Chang CH, Horton J, Schoenfeld D, Salazer O, Perez-Tamayo R, Kramer S, et al. Comparison of postoperative radiotherapy and combined postoperative radiotherapy and chemotherapy in the multidisciplinary management of malignant gliomas. A joint Radiation Therapy Oncology Group and Eastern Cooperative Oncology Group study. Cancer 1983; 52: 997-1007.

25. Chan JL, Lee SW, Fraass BA, Normolle DP, Greenberg HS, Junck LR, et al. Survival and failure patterns of high-grade gliomas after three-dimensional conformal radiotherapy. J Clin Oncol 2002; 20: 1635-42.

26. Selker RG, Shapiro WR, Burger P, Blackwood MS, Arena VC, Gilder JC, et al. The Brain Tumor Cooperative Group NIH Trial 87-01: a randomized comparison of surgery, external radiotherapy, and carmustine versus surgery, interstitial radiotherapy boost, external radiation therapy, and carmustine. Neurosurgery 2002; 51: 343-57.

27. Souhami L, Seiferheld W, Brachman D, Podgorsak EB, Werner-Wasik M Lustig R, et al. Randomized comparison of stereotactic radiosurgery followed by conventional radiotherapy with carmustine to conventional radiotherapy with carmustine for patients with glioblastoma multiforme: Report of Radiation Therapy Oncology Group 93-05 protocol. Int J Radiat Oncol Biol Phys 2004; 60: 853-60. doi: 10.1016/j.jprobp.2004.04.011

28. Werner-Wasik M, Scott CB, Nelson DF, Gaspar LE, Murray KJ, Fischbach $J A$, et al. Final report of a phase I/II trial of hyperfractionated and accelerated hyperfractionated radiation therapy with carmustine for adults with supratentorial malignant gliomas. Radiation Therapy Oncology Group Study 83-02. Cancer 1996; 77: 1535-43.

29. Badiyan SN, Markovina S, Simpson JR, Robinson CG, DeWees T, Tran DD, et al. Radiation therapy dose escalation for glioblastoma multiforme in the era of temozolomide. Int J Radiat Oncol Biol Phys 2014; 90: 877-85. doi: 10.1016/j.ijrobp.2014.07.014

30. Kelly PJ, Daumas-Duport C, Scheithauer BW, Kall BA KD. Stereotactic histologic correlations of computed tomography- and magnetic resonance imaging-defined abnormalities in patients with glial neoplasms. Mayo Clin Proc 1987; 62: 450-9.

31. Dunet V, Pomoni A, Hottinger A, Nicod-Lalonde M, Prior JO. Performance of 18 F-FET versus 18 F-FDG-PET for the diagnosis and grading of brain tumors: systematic review and meta-analysis. Neuro Oncol 2016; 18: 426-34. doi: $10.1093 /$ neuonc/nov148

32. Albert NL, Weller M, Suchorska B, Galldiks N, Soffietti R, Kim MM, et al Response assessment in Neuro-Oncology working group and European Association for Neuro-Oncology recommendations for the clinical use of PET imaging in gliomas. Neuro Oncol 2016; 18: 1199-208. doi: 10.1093/ neuonc/now058

33. Whitfield GA, Kennedy SR, Djoukhadar IK, Jackson A. Imaging and target volume delineation in glioma. Clin Oncol (R Coll Radiol) 2014; 26: 364-76. doi: 10.1016/j.clon.2014.04.026

34. Pafundi DH, Laack NN, Youland RS, Parney IF, Lowe VJ, Giannini C, et al. Biopsy validation of 18 F-DOPA PET and planning and radiotherapy target delineation: results of a prospective pilot study. Neuro Oncol 2013; 15: 1058-67. doi: 10.1093/neuonc/not002

35. Mills SJ, Du Plessis D, Pal P, Thompson G, Buonacorrsi G, Soh C, et al. Mitotic activity in glioblastoma correlates with estimated extravascular extracellular space derived from dynamic contrast-enhanced MR imaging. Am J Neuroradiol 2016; 37: 811-7. doi: 10.3174/ajnr.A4623

36. Brindle KM, Izquierdo-García JL, Lewis DY, Mair RJ, Wright AJ. Brain tumor imaging. J Clin Oncol 2017; 35: 2432-8. doi: 10.1200/JCO.2017.72.7636

37. Niyazi M, Geisler J, Siefert A, Schwarz SB, Ganswindt U, Garny S, et al. FETPET for malignant glioma treatment planning. Radiother Oncol 2011; 99: 44-8. doi: 10.1016/j.radonc.2011.03.001

38. Sulman EP, Ismaila N, Armstrong TS, Tsien C, Batchelor TT, Cloughesy T, et al. Radiation therapy for glioblastoma: American Society of Clinical Oncology Clinical Practice Guideline Endorsement of the American Society for Radiation Oncology Guideline. J Clin Oncol 2017; 35: 361-9. doi: 10.1200/ JCO.2016.70.7562

39. Niyazi M, Brada M, Chalmers AJ, Combs SE, Erridge SC, Fiorentino A, et al. ESTRO-ACROP guideline "target delineation of glioblastomas." Radiother Oncol 2016; 118: 35-42. doi: 10.1016/j.radonc.2015.12.003

40. Minniti G, Amelio D, Amichetti M, Salvati M, Muni R, Bozzao A, et al. Patterns of failure and comparison of different target volume delineations in patients with glioblastoma treated with conformal radiotherapy plus concomitant and adjuvant temozolomide. Radiother Oncol 2010; 97: 37781. doi: 10.1016/j.radonc.2010.08.020 
41. Louis DN, Perry A, Reifenberger G, von Deimling A, Figarella D, Webster B, et al. The 2016 World Health Organization classification of tumors of the central nervous system: a summary. Acta Neuropathol 2016; 131: 803-20. doi: 10.1007/s00401-016-1545-1

42. Chan MF, Schupak K, Burman C, Chui C-S, Ling CC. Comparison of intensity-modulated radiotherapy with three-dimensional conformal radiation therapy planning for glioblastoma multiforme. Med Dosim 2003; 28: 261-5. doi: 10.1016/j.meddos.2003.08.004

43. Panet-Raymond V, Souhami L, Roberge D, Kavan P, Shakibnia L, Muanza T, et al. Accelerated hypofractionated intensity-modulated radiotherapy with concurrent and adjuvant temozolomide for patients with glioblastoma multiforme: a safety and efficacy analysis. Int J Radiat Oncol Biol Phys 2009; 73: 473-8. doi: 10.1016/j.jijrobp.2008.04.030

44. Shenouda G, Souhami L, Petrecca K, Owen S, Panet-Raymond V, Guiot $\mathrm{M}-\mathrm{C}$, et al. A phase 2 trial of neoadjuvant temozolomide followed by hypofractionated accelerated radiation therapy with concurrent and adjuvant temozolomide for patients with glioblastoma. Int J Radiat Oncol Biol Phys 2017; 97: 487-94. doi: 10.1016/j.jjrobp.2016.11.006

45. Ataman F, Poortmans P, Stupp R, Fisher B, Mirimanoff RO. Quality assurance of the EORTC 26981/22981; NCIC CE3 intergroup trial on radiotherapy with or without temozolomide for newly-diagnosed glioblastoma multiforme: the individual case review. Eur J Cancer 2004; 40: 1724-30. doi: 10.1016/j. ejca.2004.03.026

46. Kazda T, Jancalek R, Pospisil P, Sevela O, Prochazka T, Vrzal M, et al. Why and how to spare the hippocampus during brain radiotherapy: the developing role of hippocampal avoidance in cranial radiotherapy. Radiat Oncol 2014; 9: 139. doi: 10.1186/1748-717X-9-139

47. Gondi V, Hermann BP, Mehta MP, Tomé WA. Hippocampal dosimetry predicts neurocognitive function impairment after fractionated stereotactic radiotherapy for benign or low-grade adult brain tumors. Int J Radiat Oncol Biol Phys 2013; 85: 348-54. doi: 10.1016/j.jijrobp.2012.11.031

48. Gzell C, Back M, Wheeler H, Bailey D, Foote M. Radiotherapy in glioblastoma: the past, the present and the future. Clin Oncol (R Coll Radiol) 2017; 29: 15-25. doi: 10.1016/j.clon.2016.09.015

49. Smith AW, Mehta MP, Wernicke AG. Neural stem cells, the subventricular zone and radiotherapy: implications for treating glioblastoma. J Neurooncol 2016; 128: 1-10. doi: 10.1007/s11060-016-2123-z

50. Chen L, Chaichana KL, Kleinberg L, Ye X, Quinones-Hinojosa A, Redmond K. Glioblastoma recurrence patterns near neural stem cell regions. Radiother Oncol 2015; 116: 294-300. doi: 10.1016/j.radonc.2015.07.032

51. Khalifa J, Tensaouti F, Lusque A, Plas B, Lotterie J-A, Benouaich-Amiel A, et al. Subventricular zones: new key targets for glioblastoma treatment. Radiat Oncol 2017; 12: 67. doi: 10.1186/s13014-017-0791-2

52. Nourallah B, Digpal R, Jena R, Watts C. Irradiating the subventricular zone in glioblastoma patients: is there a case for a clinical trial? Clin Oncol ( $R$ Coll Radiol) 2017; 29: 26-33. doi: 10.1016/j.clon.2016.09.005

53. Pospisil P, Kazda T, Hynkova L, Bulik M, Dobiaskova M, Burkon $P$, et al. Post-WBRT cognitive impairment and hippocampal neuronal depletion measured by in vivo metabolic MR spectroscopy: results of prospective investigational study. Radiother Oncol 2017; 122: 373-9. doi: 10.1016/j. radonc.2016.12.013

54. Flechl B, Sax C, Ackerl M, Crevenna R, Woehrer A, Hainfellner J, et al. The course of quality of life and neurocognition in newly diagnosed patients with glioblastoma. Radiother Oncol 2017; 125: 228-33. doi: 10.1016/j. radonc.2017.07.027

55. Weller M, Roth $P$, Preusser M, Wick W, Reardon DA, Platten M, et al. Vaccine-based immunotherapeutic approaches to gliomas and beyond. Nat Rev Neurol 2017; 13: 363-74. doi: 10.1038/nrneurol.2017.64

56. Sampson JH, Maus MV, June CH. Immunotherapy for brain tumors. J Clin Oncol 2017; 35: 2450-6. doi: 10.1200/JCO.2017.72.8089

57. Reznik E, Smith AW, Taube S, Mann J, Yondorf MZ, Parashar B, et al. Radiation and immunotherapy in high-grade gliomas. Am J Clin Oncol 2017 Sep 12. doi: 10.1097/COC.0000000000000406. [Epub ahead of print] 